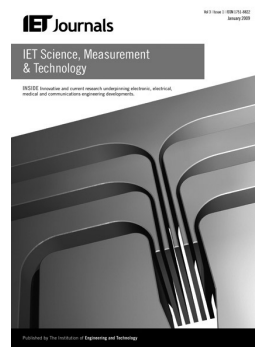

ISSN 1751-8822

\title{
Numerical and experimental study of the effects of load and distance variation on wireless power transfer systems using magnetically coupled resonators
}

\author{
Mihai Dragos Rotaru', Robin Tanzania ${ }^{2}$, Raed Ayoob ${ }^{1}$, Tan Yen Kheng ${ }^{2}$, Jan K. Sykulski ${ }^{1}$ \\ ${ }^{1}$ University of Southampton, Southampton, UK \\ ${ }^{2} E R I A N$, Nanyang Technological University, Singapore, Singapore \\ E-mail:mr@esc.soton.ac.uk
}

\begin{abstract}
This work investigates a series resonant circuit designed to wirelessly transfer power to charge an electrical vehicle battery. A typical approach assumes the load connected to the power transfer system to be constant and then the wireless link efficiency is studied. In practical engineering applications, however, the load and the distance between coils will vary and the efficiency may strongly depend on these variations. The efficiency will also be affected by the presence of massive conducting or shielding structures in the proximity of the wireless system. Here, the authors study these effects with the help of an equivalent circuit extracted from a full wave simulation and correlated with measured results. The authors demonstrate that by changing the load resistance the efficiency of the system can be improved, even for a large separation between the two magnetically coupled resonators; however, the maximum efficiency point may not correspond to the maximum power that can be handled by the system. They then analyse the primary and secondary voltages and currents in support of the above findings.
\end{abstract}

\section{Introduction}

Recent years have seen growing interest in wireless power transfer (WPT) technologies. The inductively coupled power transfer systems have been in use for some time for different applications [1-4]; unfortunately the efficiency of such systems decreases dramatically when the separation between the two coupled coils is increased. In 2006 a much more efficient method exploiting magnetically resonant coupling was proposed and confirmed experimentally in 2007 [5], allowing higher efficiency to be achieved at much larger air gaps. The WPT system usually consists of two coils that are brought to resonance with the help of capacitors. Different system structures have been put forward [6] and their capability of WPT analysed. It has been shown that a resonant circuit where the coil and capacitor are connected in series is suitable for higher power systems required for charging wirelessly batteries in electric vehicles $[7,8]$.

In this study a wireless power system working at $100 \mathrm{kHz}$ and capable of handling up to $2 \mathrm{~kW}$ of power has been designed, modelled, analysed and tested. The system relies on a near field coupling mechanism which is non-radiative, operates at distances well below the wavelength of the electromagnetic wave being transmitted and can efficiently transfer power up to $300 \mathrm{~mm}$. However, the efficiency is highly sensitive to changes to the distance between the coils, the value of the connected load and may also be affected by the shielding structures introduced so that the set-up complies with ICNIRP Guidelines [9]. From a full finite element model of the resonating coupled coils an equivalent circuit has been extracted, and both are then used to analyse the effect of the distance, load, as well as the shielding on the power transfer system. The circuit model is also used to aid the design process as it is much faster than the full three-dimensional (3D) solution. The simulations have been verified by measurements performed on a similar system.

\section{3D full wave electromagnetic model of the WPT system}

A 3D model of the coils has been built and solved using commercial software assuming: coil diameter $300 \mathrm{~mm}$, conductor diameter $2.6 \mathrm{~mm}$ and insulated wire diameter 3.6 $\mathrm{mm}$. The coils have 28 turns; however, they are distributed unevenly in two layers (Fig. 1); one layer has 21 turns whereas the second layer has 7 turns. Two capacitors of $22.6 \mathrm{nF}$ each have been connected in series with the coil. In the experimental setup of the same system, strings of Class $1 \mathrm{C} 0 \mathrm{G} / \mathrm{NP} 0$ ceramic capacitors have been used to achieve the desired capacitances.

The 3D model of one coil was initially solved in frequency domain. The excitation assumed was a current source with an impedance of $50 \Omega$. The source produces a $1 \mathrm{~W}$ input power which enables the calculation of scattering $(S)$ parameters, with the S11 parameter found for a frequency sweep from $\mathrm{DC}$ up to $1 \mathrm{MHz}$. One coil with two series capacitors has a 

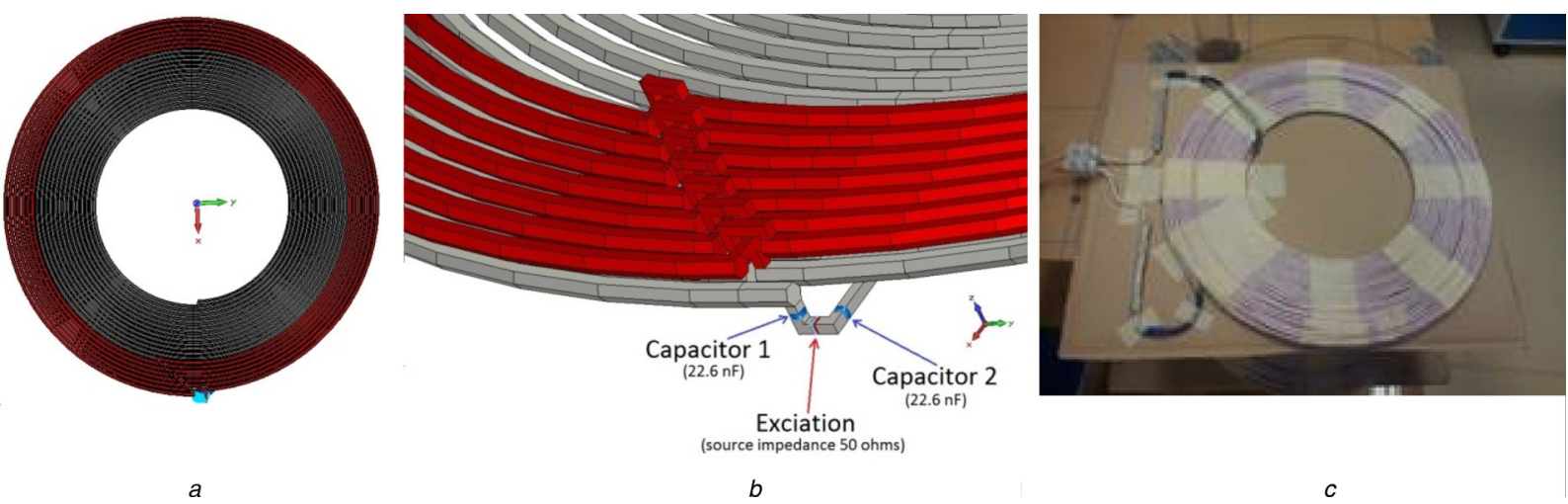

Fig. 1 Resonant coils of the WPT system

$a$ Top view of the two-layer coil

$b$ Close-up view of the two layers

$c$ Top view of the experimental set-up

clear resonance at $96.7 \mathrm{kHz}$ as shown in Fig. 2, where the phase against frequency is plotted. Using this information and basic circuit theory the inductance of the coil may be calculated. For a series resonant circuit the resonant frequency can thus be calculated as

$$
f_{\mathrm{res}}=\frac{1}{2 \pi \sqrt{L C}}
$$

The inductance of the coil computed using (1) is $240.7 \mu \mathrm{H}$. As a reference the measured value of the inductance was $235 \mu \mathrm{H}$. The resistance of the coil was estimated from its geometry assuming it was made using a copper Litz wire with 210 strands (type AWG33). Each strand of wire has a diameter of $0.18 \mathrm{~mm}$. For this set-up the skin effect will become important only for frequencies above $1 \mathrm{MHz}$, hence it was ignored in the calculation. The computed resistance was $0.035 \Omega$.

Following the single coil analysis, two identical coils were simulated in 3D. The distance between the coils was varied and the results in terms of $\mathrm{S}$ parameters used to extract the coupling factor between the two coils. To reduce the computational burden only five distances were used in the 3D full wave model: 20, 50, 100, 150 and $200 \mathrm{~mm}$. The magnitudes of the $\mathrm{S}$ parameters (S11 and S12) are presented in Fig. 3.

The coupling factor, $K$, was extracted with the help of a circuit simulator. An equivalent circuit, as shown in Fig. 4, was derived and simulated. Using a frequency domain solver the solution in terms of the $\mathrm{S}$ parameters was obtained. The coupling factor between the two coils was adjusted so that the $\mathrm{S}$ parameter results obtained from the circuit simulator matched those obtained from the full wave solver. Fig. 5 compares the solutions obtained with the full wave solver and from the equivalent circuit shown in Fig. 4 in terms of the magnitude and phase of the scattering parameters of the system. It will be noted that the two sets of results agree very well.

Using this methodology the coupling factor was extracted for all five cases considered and the results are summarised in Table 1. The extracted results were also validated by measurements taken using an experimental wireless charging setup (Fig. 6). As expected the coupling factor decreases as the distance between the two coils increases. A polynomial function was used to fit the data from Table 1 . A cubic polynomial was found to fit the data very well so that the coupling factor can be easily calculated using this interpolation function for any distance between the coils in the range between 20 and $200 \mathrm{~mm}$. If data needs to be extrapolated outside this range extra full wave simulations will be required and a new polynomial fitted. However, for the work presented here, the discussion was limited to distances between 20 and $200 \mathrm{~mm}$.

The full wave solution of the WPT system has also provided useful insight regarding the distribution of magnetic field around the coils. As a frequency sweep was applied the field distribution around the system was obtained for several frequencies. The magnetic field intensity along a line starting in the middle of one coil and extending $300 \mathrm{~mm}$ from that coil, passing through the middle of the other coil, for different frequencies is shown in Fig. 7. It is clear that the field intensity at resonance is
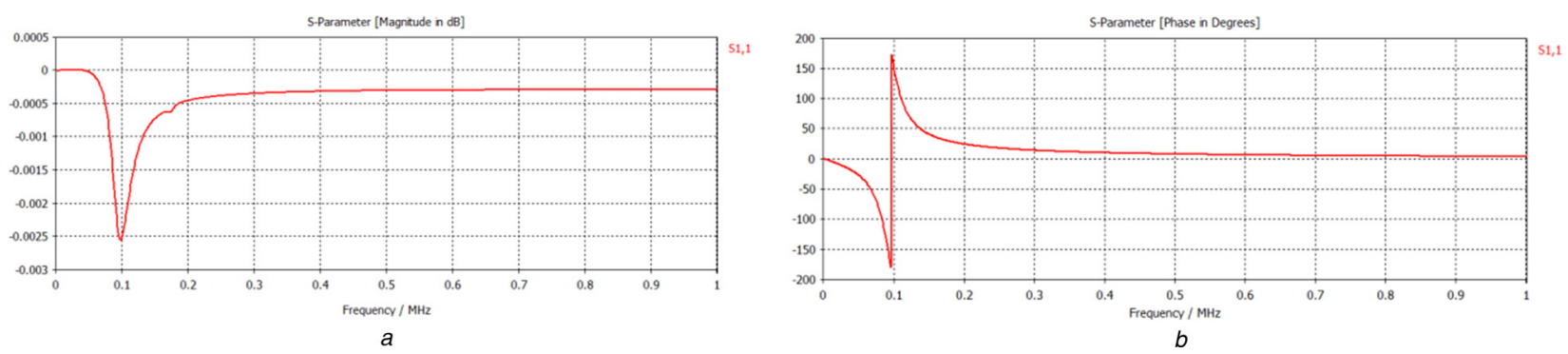

Fig. $2 S$ parameter for the coil-capacitor series arrangement $a$ Magnitude $b$ Phase 


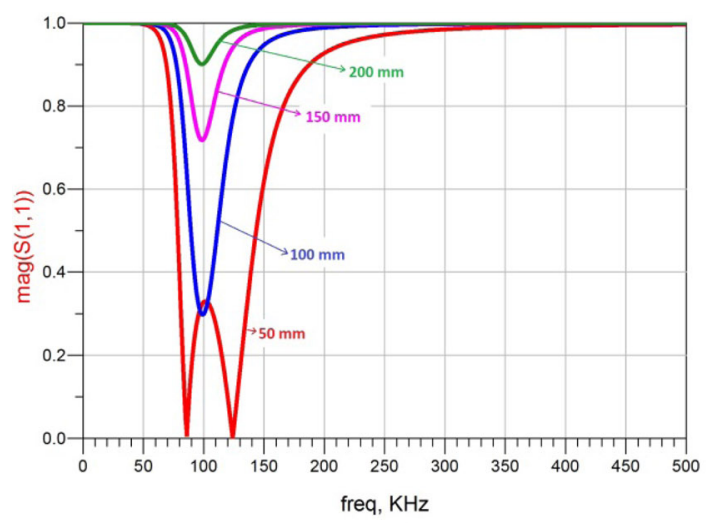

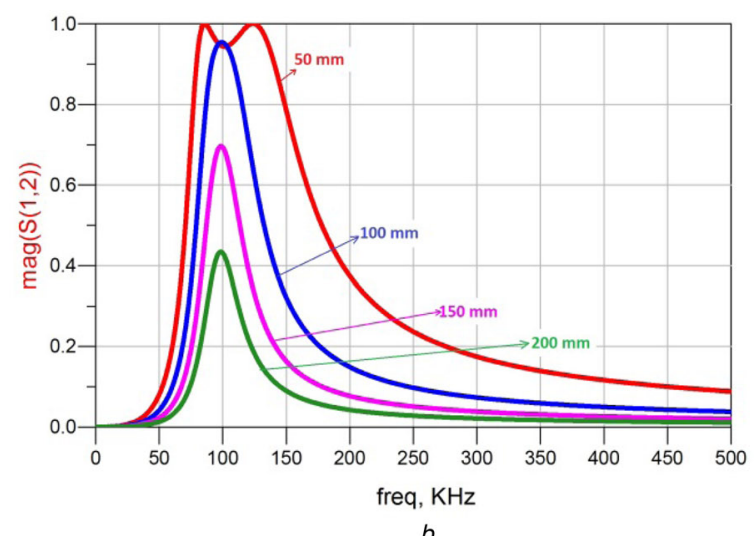

Fig. 3 S parameters for the magnetically coupled coils

$a$ Magnitude of $\mathrm{S} 11$

$b$ Magnitude of S21

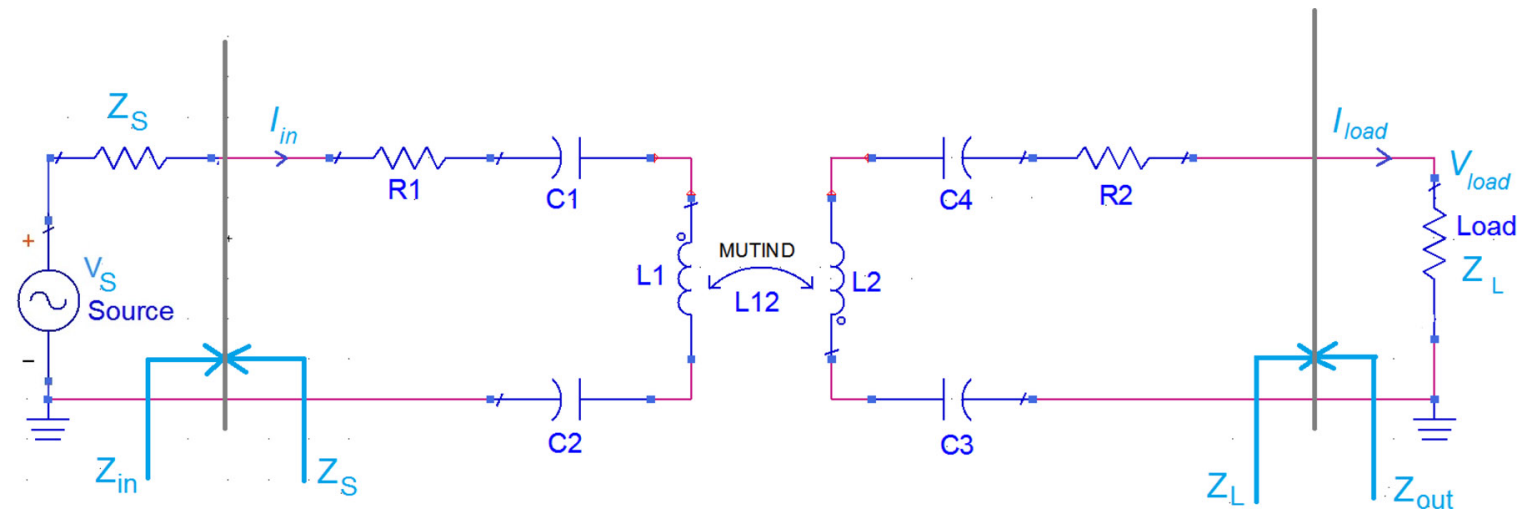

Fig. 4 Equivalent circuit of the magnetically coupled coils
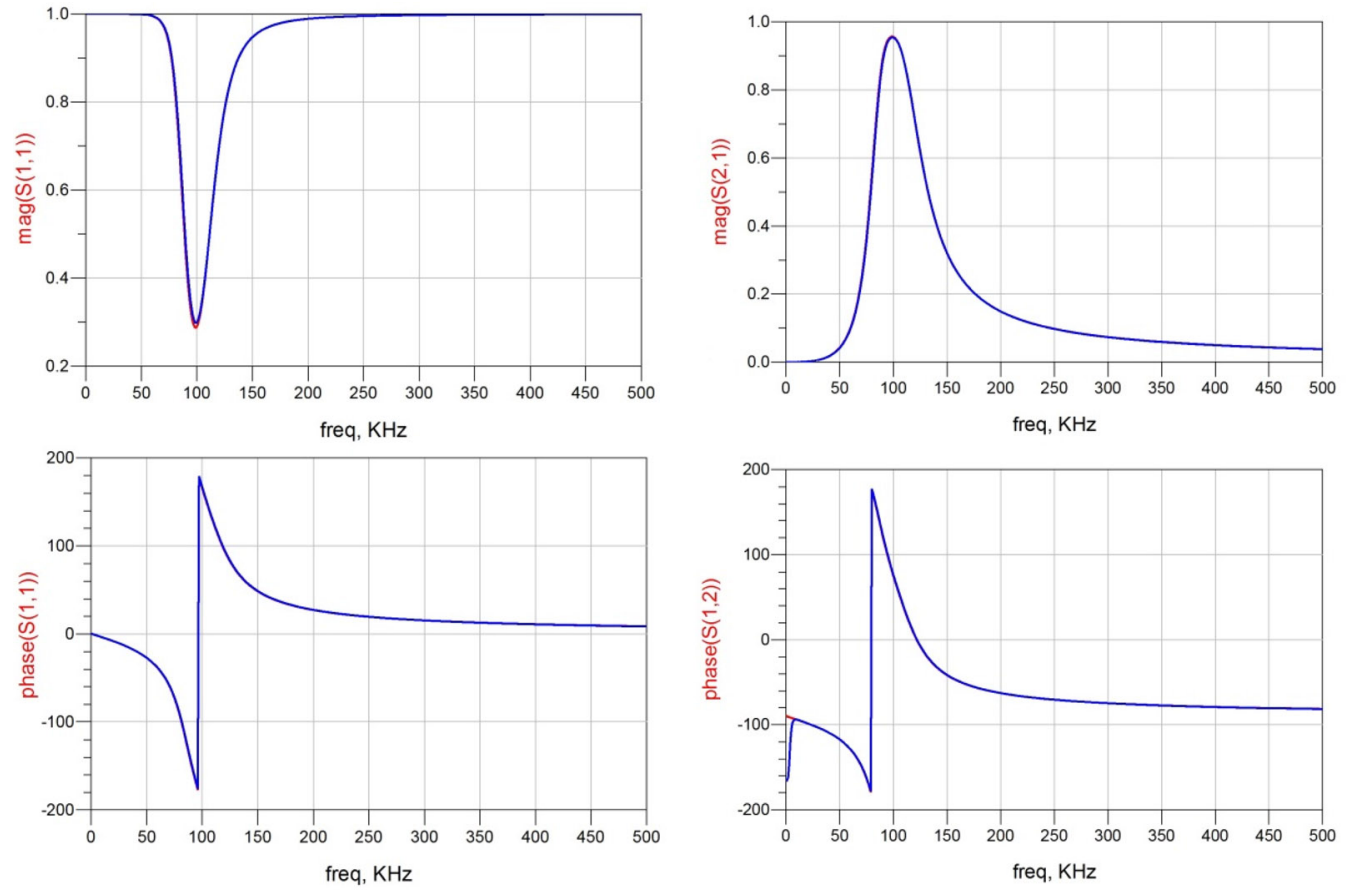

$b$

Fig. 5 Comparison between full wave and equivalent circuit solutions for the $100 \mathrm{~mm}$ gap case

$a$ Magnitude and phase of S11

$b$ Magnitude and phase of S12 
Table 1 Extracted coupling factor

\begin{tabular}{lc}
\hline Distance, $\mathrm{mm}$ & Coupling factor $K$ \\
\hline 20 & 0.736 \\
50 & 0.4800 \\
100 & 0.2520 \\
150 & 0.1395 \\
200 & 0.0780 \\
the polynomial function that has been fitted to the above data \\
are $K(x)=-(1.305 e-7)^{*} x^{3}+(6.623 e-5)^{*} x^{2}-(-1.243 e-2)^{*} x+$ \\
0.957 \\
\hline
\end{tabular}

much higher than for non-resonant frequencies. From Figs 7 and 8 it is also apparent that for non-resonant frequencies the variation of the field away from the excited coil is different as compared with the resonant frequency. At resonance, as the field approaches the load coil levels instead of decreasing, this is mainly because of the induced current in the load coil which produces its own field adding to the source field.

Distributions of magnetic field intensity in the $y z$-plane, obtained from the $3 \mathrm{D}$ model solution, are also provided in Fig. 8 for three different frequencies: 20,100 and $200 \mathrm{kHz}$. As expected the intensity of the magnetic field, and thus the density of the field lines, are much higher around the resonance frequency (Fig. $8 b$ ). It is also clear from these plots that the field around the secondary coil is much larger at resonance than when out of resonance. In Fig. 8 coil 2 is excited while coil 1 is receiving. The case illustrated in Fig. 8 is for a distance of $150 \mathrm{~mm}(K=0.1395)$ between the two coils, hence there will be no frequency splitting (no over-coupling). The field plot would be different in the case of an over-coupled system; it was not the intention of this work, however, to study the field distributions in any detail and under different conditions.

\section{Analysis and discussion of the equivalent circuit of WPT system}

The WPT system can be described and analysed conveniently using a two-port network theory. As shown graphically in Fig. 4, the wireless system is fed at port one by the power source, whereas the second port is connected to the load. Using this approach one can extract the scattering parameters of the network. In this case the efficiency of the wireless link can be described conveniently by the magnitude of S21. S21 can be used to determine the insertion loss of the channel between ports 1 and 2 when the output is terminated by a matching load. In most cases the termination load is assumed to be $50 \Omega$. Moreover, S21 can be used to calculate the transducer gain, given by $\left|S_{21}\right|^{2}$, which is defined as the power delivered to the load over the available power from the source when both the source and load impedances are perfectly matched.

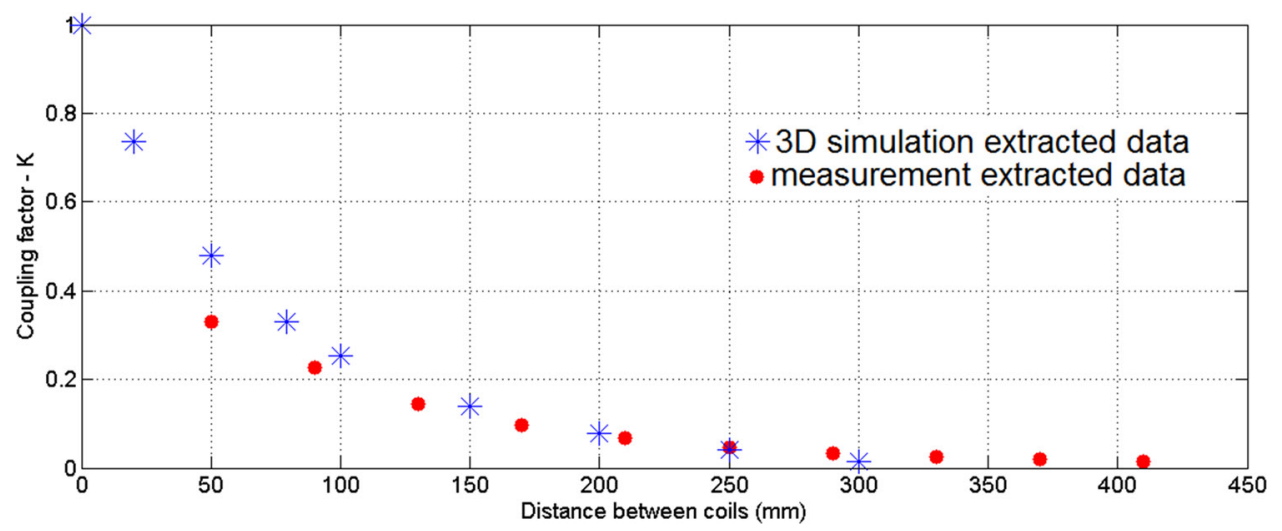

Fig. 6 Comparison of measured and simulated values of the coupling factor $K$

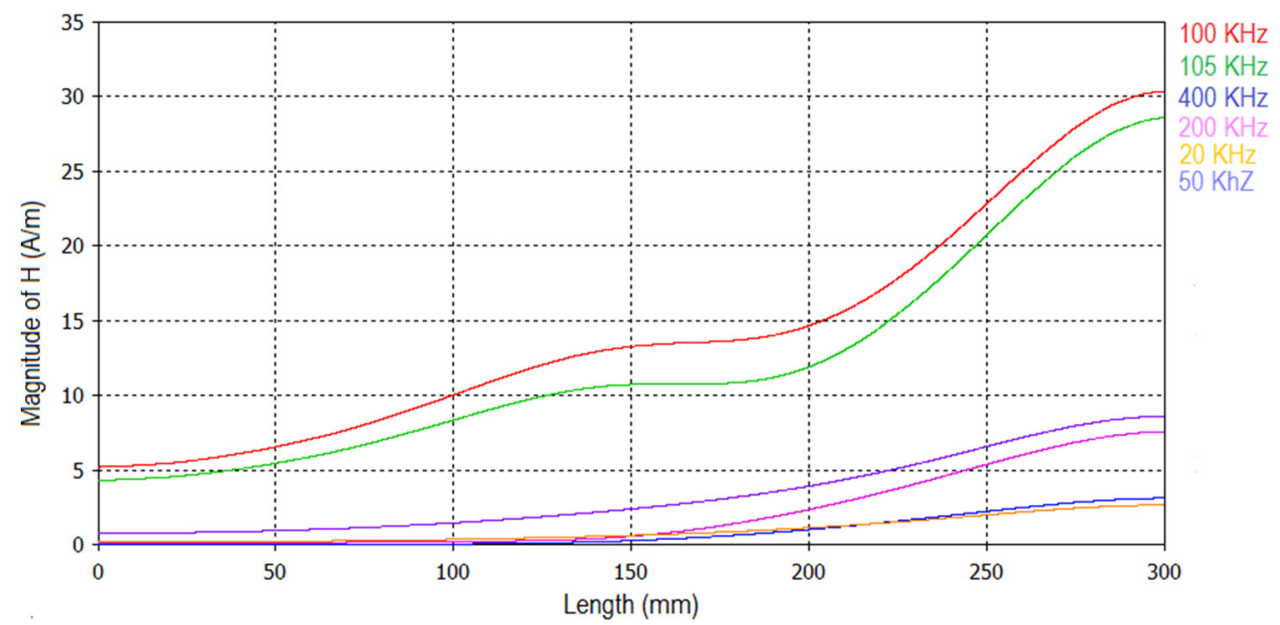

Fig. 7 Magnetic field intensity distribution along a $300 \mathrm{~mm}$ line ending in the middle of the primary coil and going through the middle of the secondary coil (distance between coils is $150 \mathrm{~mm}$ ) 


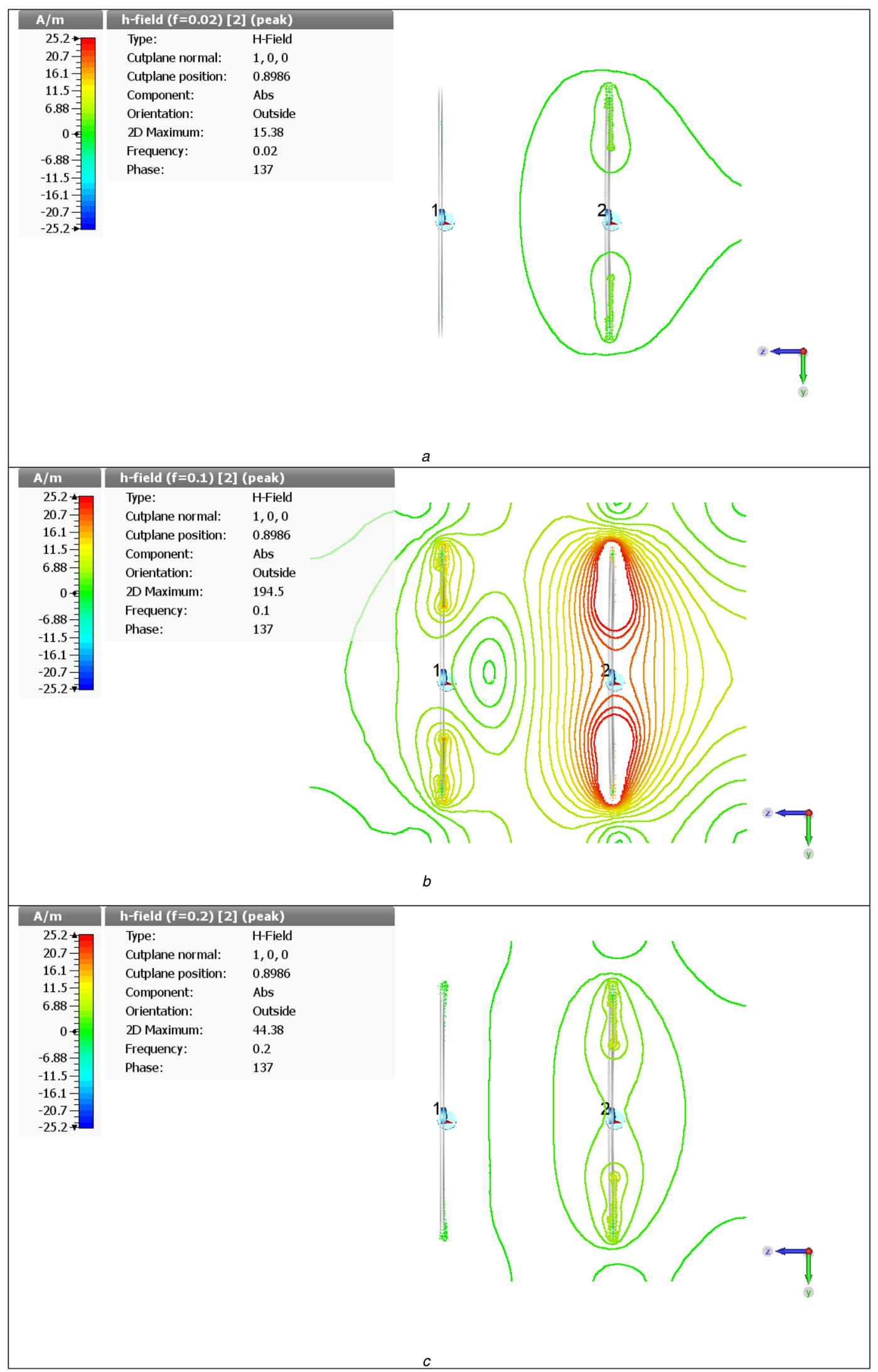

Fig. 8 Magnetic field intensity distribution at

a 20

$b 100$

c $200 \mathrm{kHz}$ around the WPT system when the coils are $150 \mathrm{~mm}$ apart 


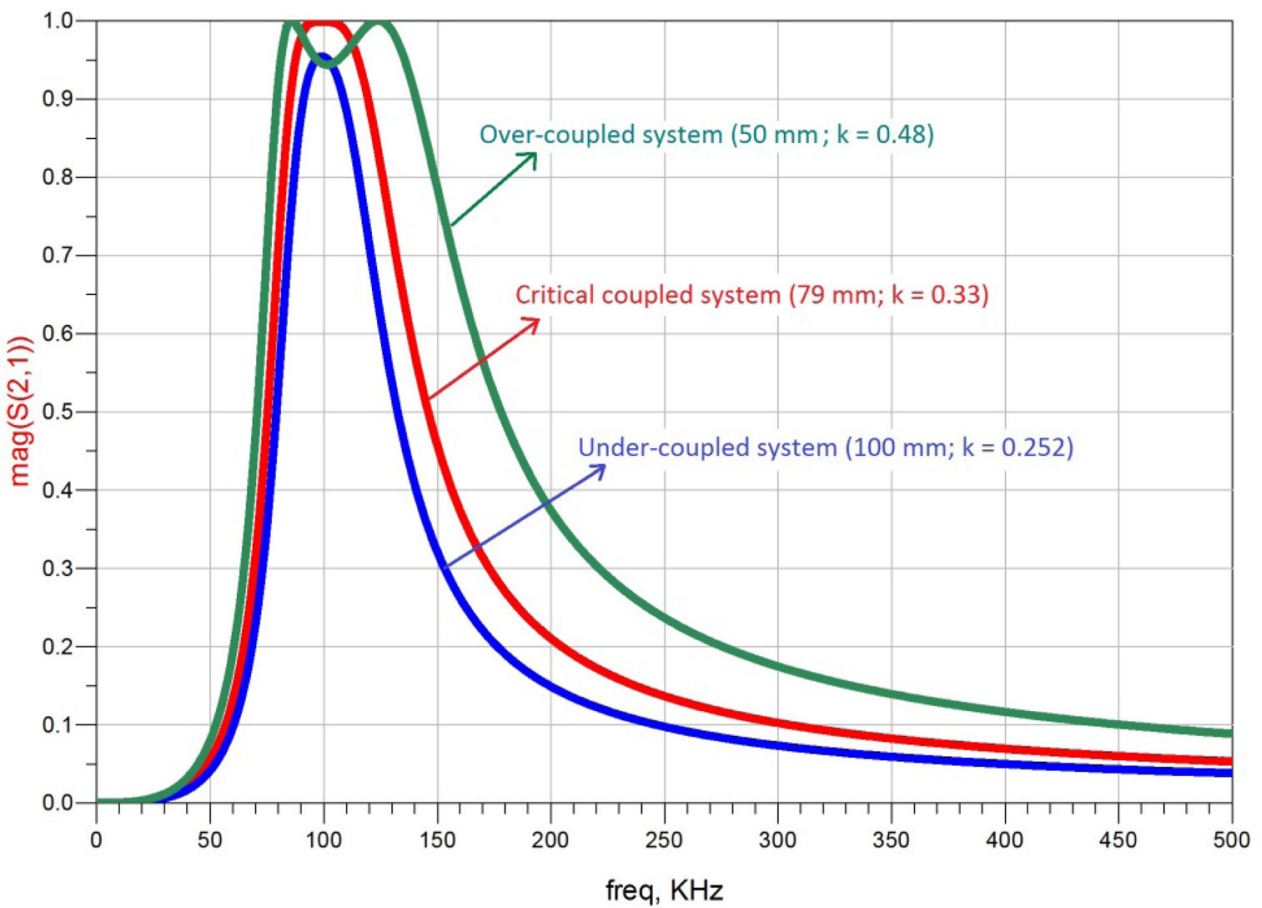

Fig. 9 Magnitude of S21 for over-, critically-and under-coupled systems

For the system studied here S21 can be expressed in terms of the circuit components (Fig. 4) using (2), where $Z_{\mathrm{S}}$ is the source impedance, $Z_{\mathrm{L}}$ is the load impedance, $K$ is the coupling factor between the two coils, $R_{1}, L_{1}$ and $C_{11}\left(C_{11}\right.$ $\left.=C_{1} C_{2} /\left(C_{1}+C_{2}\right)\right)$ are the circuit components associated with the source loop, whereas $R_{2}, L_{2}$ and $C_{22}\left(\mathrm{C}_{22}=C_{3} C_{4} /\right.$ $\left.\left.\left(C_{3}+C_{4}\right)\right)\right)$ are the components in the load loop.

Using this approach the WPT system described in Section 2 has been analysed. The results in terms of the magnitude of scattering parameters, when the distance between coils is varied, are shown in Fig. 3. The source and load impedances for all cases were assumed to be $50 \Omega$. It is clear from the results that when two coils are $50 \mathrm{~mm}$ apart the system is over-coupled; the frequency splitting phenomena, well described in [7], is evident. Two resonance peaks are typical for such cases and are clearly visible in Fig. 3. The maximum transfer efficiency (mag $(\mathrm{S} 21)=1)$ is achievable at both resonant peaks. When the distance is increased to $100 \mathrm{~mm}$ the system becomes under-coupled and $\operatorname{mag}(\mathrm{S} 21)<1$, which implies that the system will not be transferring power at its maximum efficiency. For the system analysed here the critically coupled point, as defined in [7], is reached when the coupling factor is $K=0.33$. This value has been obtained using the equivalent circuit shown in Fig. 4 and the definition of the critical coupling point [7] (see Fig. 9). Using the cubic polynomial function (2) the distance at which this critical point occurs can be approximated; in this case the distance is $79 \mathrm{~mm}$. At this distance the WPT system will transfer power at its maximum efficiency at one frequency which is the resonant frequency of the coils. For any distance larger than $79 \mathrm{~mm}$ the efficiency of the system will drop below its maximum as illustrated in Fig. 8.

The WPT system described in [7] had the load and source impedance fixed and the critical coupling was defined in terms of the distance between the two coils. By varying the distance between the coils the coupling factor can be adjusted so that the maximum power transfer is achieved. However, the magnitude of S21, which can be used to judge the efficiency of the wireless link, does not depend on $K$ only but also on other factors. The load and source impedances are factors that can be modified externally; hence they could be useful in controlling and improving the wireless link efficiency. To illustrate the effect of the load impedance on the efficiency of the WPT system a simple experiment using the equivalent circuit shown in Fig. 4 was performed. The coupling factor, hence the distance between the two coils, were fixed and the real part of the load impedance was varied. To simplify the analysis the imaginary part of the load impedance was assumed to be zero, hence only the resistive part of the impedance was considered in the following examples. The experiment was conducted for three cases, namely a critically coupled system, an over-coupled system and an under-coupled system, respectively. These systems were defined for a $50 \Omega$ source and load impedances as a reference.

For the first case of this numerical experiment, the starting point was an over-coupled system; as already

$$
\begin{aligned}
S_{21}(\omega) & =\frac{2 j \omega K \cdot \sqrt{L_{1} L_{2}} \cdot \sqrt{Z_{\mathrm{s}} Z_{\mathrm{L}}}}{\left(Z_{\mathrm{s}}+R_{1}+j \omega L_{1}+\left(1 /\left(j \omega C_{11}\right)\right)\right)\left(Z_{\mathrm{L}}+R_{2}+j \omega L_{2}+\left(1 /\left(j \omega C_{22}\right)\right)\right)+K^{2} \omega^{2} L_{1} L_{2}} \\
& =\frac{2 j \omega L_{12} \cdot \sqrt{Z_{\mathrm{s}} Z_{\mathrm{L}}}}{\left(Z_{\mathrm{s}}+R_{1}+j \omega L_{1}+\left(1 /\left(j \omega C_{11}\right)\right)\right)\left(Z_{\mathrm{L}}+R_{2}+j \omega L_{2}+\left(1 /\left(j \omega C_{22}\right)\right)\right)+L_{12}^{2} \omega^{2}}
\end{aligned}
$$



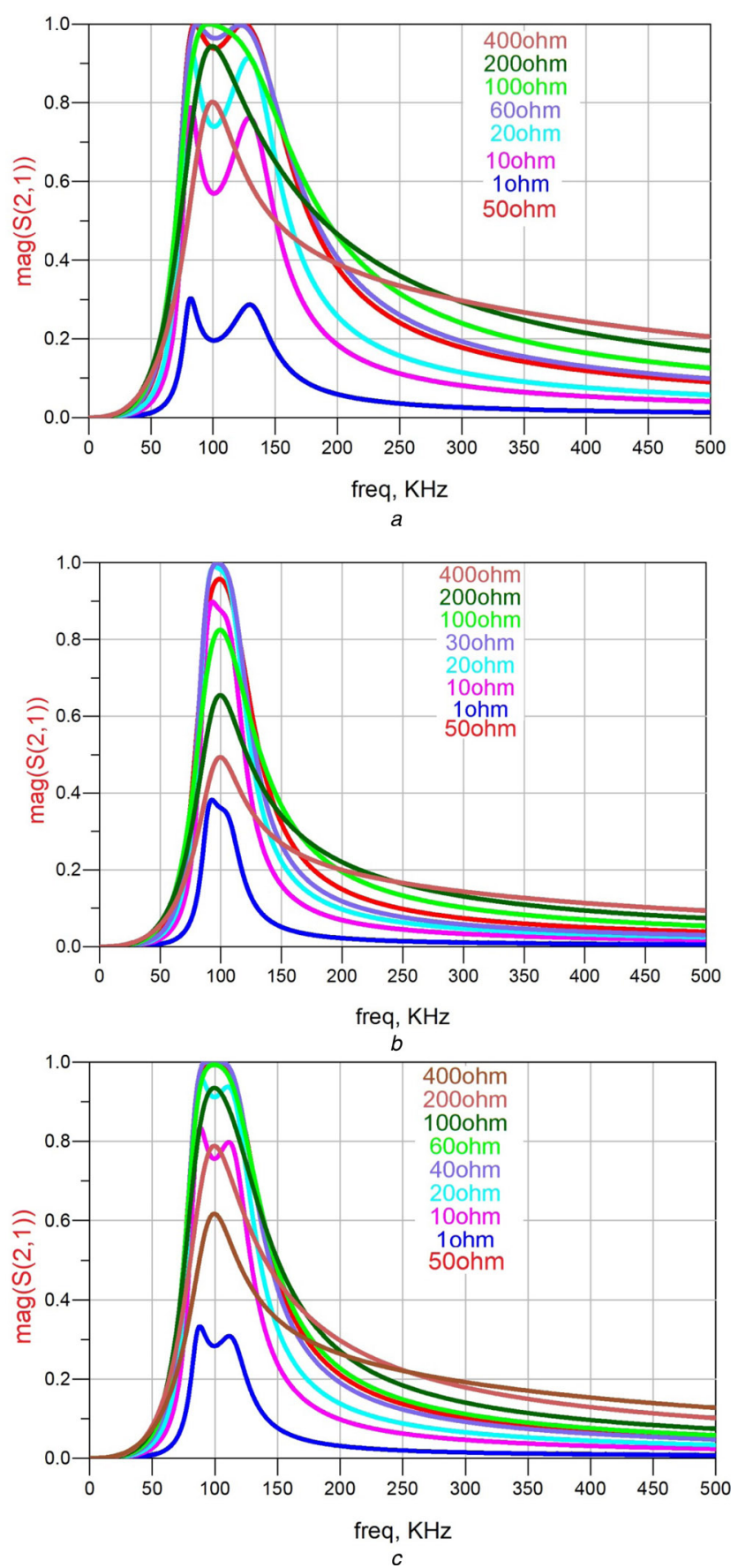

Fig. 10 Magnitude of S21 variation for different load conditions when the system is

$a$ Over-coupled (50 $\mathrm{mm}$ distance between the coils)

$b$ Under-coupled (100 $\mathrm{mm}$ distance between the coils)

$c$ Critically coupled ( $79 \mathrm{~mm}$ between the coils)

mentioned the load and source impedances for this case were $50 \Omega$.

With the load impedance varied from 1 to $400 \Omega$, with the source impedance fixed at $50 \Omega$ and the distance between coils fixed to $50 \mathrm{~mm}(K=0.48)$, the change in S21 was observed. The magnitude of S21 is presented in Fig. 10; as can be seen in Fig. $10 a$ the system is initially in an over-coupled state. However, increasing the load impedance from 50 to $100 \Omega$ shifts the WPT to a state that could be described as critically coupled. Increasing the load further makes the WPT under-coupled.
Similar behaviour may be observed when the load connected to the WPT system is varied but the starting point is a critical coupled state, or an under-coupled state, as shown graphically in Figs $10 b$ and $c$. Following from these results it might be argued that the region in which the system is critically coupled could be extended by changing the load impedance. Furthermore, a similar response can be obtained by varying the source impedance rather than the load impedance.

To understand further the behaviour of efficiency of the WPT system with respect to the load and distance between the two coils a surface plot of the magnitude S21 at the resonance frequency $(96.7 \mathrm{kHz})$ was constructed using (2) and is presented in Fig. 11; the source impedance $Z_{\mathrm{S}}$ is 50 $\Omega$ as before. It can be noted that the system can have very high coupling ( $>90 \%$ ) even at a large separation distance, such as $200 \mathrm{~mm}$, but only for a small load impedance. When increasing the load the distance has to be reduced in order to keep the coupling efficiency at values above $90 \%$. For load impedances up to $60 \Omega$ decreasing the distance between the coils below $100 \mathrm{~mm}$ will reduce the coupling efficiency at the resonant frequency of $96.7 \mathrm{kHz}$, as in this region the system becomes over-coupled, and to restore the maximum coupling efficiency the frequency would need to be changed, as explained in [7]. Although the maximum efficiency of the system can be tracked, using a frequency tuner coupled with a controller that follows the maximum resonant peak as the load (receiver) is moved with respect to the transmitter [7], this solution is complex and introduces additional components into the WPT system, hence reducing the overall efficiency. On the other hand, recent work [8] has shown that the highest efficiency is achieved at the natural resonant frequency $\left(\omega_{0}\right)$ rather than any of the split resonant frequencies of the magnetically coupled WPT system.

It will be helpful to return to the equivalent circuit of Fig. 4 and extract the input and output impedances $\left(Z_{\text {in }}\right.$ and $\left.Z_{\text {out }}\right)$ of the circuit

$$
\begin{aligned}
Z_{\text {in }}(\omega)= & \frac{1}{j \omega C_{11}}+j \omega L_{1} \\
& +R_{1}+\frac{\left(\omega L_{12}\right)^{2}}{j \omega L_{2}+\left(1 /\left(j \omega C_{22}\right)\right)+R_{2}+Z_{\mathrm{L}}} \\
Z_{\text {out }}(\omega)= & \frac{1}{j \omega C_{22}}+j \omega L_{2} \\
& +R_{2}+\frac{\left(\omega L_{12}\right)^{2}}{j \omega L_{1}+\left(1 /\left(j \omega C_{11}\right)\right)+R_{1}+Z_{\mathrm{S}}}
\end{aligned}
$$

At resonance both input and output impedances become purely real and, if the two loops are identical, this resonance occurs at the frequency given by (1). At resonance $\left(\omega_{0}\right)$ the two impedances $\left(Z_{\text {in }}\right.$ and $\left.Z_{\text {out }}\right)$ can be written as

$$
\begin{aligned}
& Z_{\text {in }}\left(\omega_{0}\right)=R_{1}+\frac{\left(\omega_{0} L_{12}\right)^{2}}{R_{2}+Z_{\mathrm{L}}}=R_{1}+\frac{k^{2} \omega_{0}^{2} L_{1} L_{2}}{R_{2}+Z_{\mathrm{L}}} \\
& Z_{\text {out }}\left(\omega_{0}\right)=R_{2}+\frac{\left(\omega_{0} L_{12}\right)^{2}}{R_{1}+Z_{\mathrm{S}}}=R_{2}+\frac{k^{2} \omega_{0}^{2} L_{1} L_{2}}{R_{1}+Z_{\mathrm{S}}}
\end{aligned}
$$

It is informative to plot the variation of $Z_{\text {out }}\left(\omega_{0}\right)$ with the distance between coils when $Z_{\mathrm{s}}$ is kept constant (in Fig. 12 


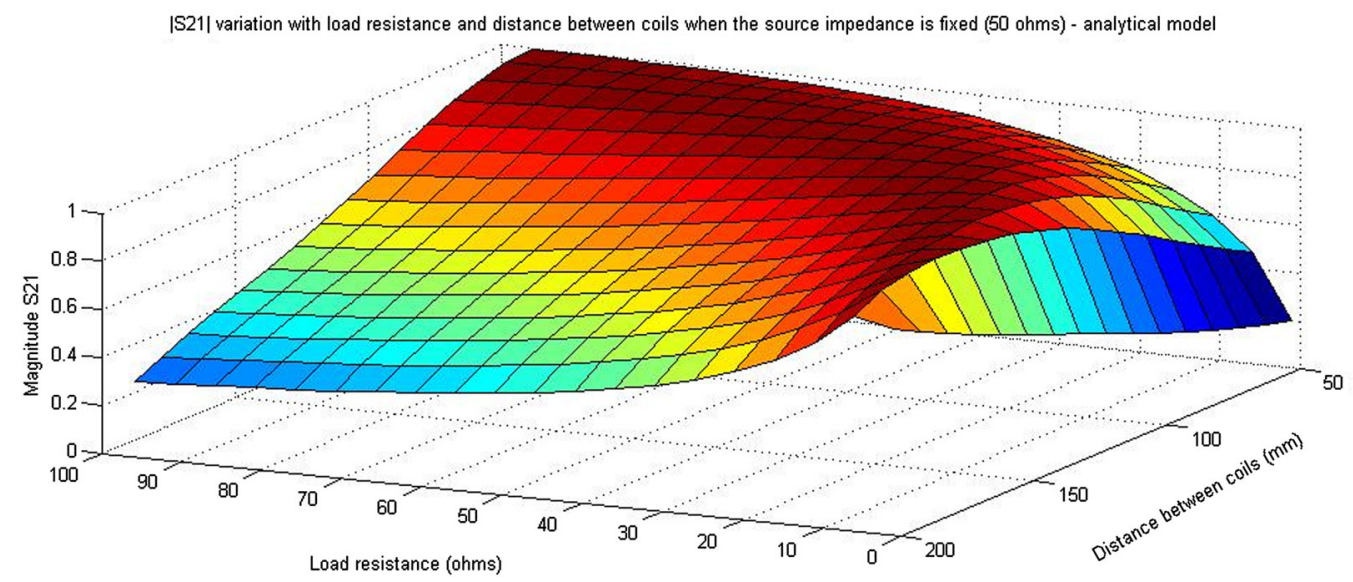

Fig. 11 Variation of S21 magnitude when the load and distance between coils is changed (the source impedance is fixed at $50 \Omega$ )

$Z_{\mathrm{s}}=50 \Omega$ ). Considering both Figs 11 and 12 it can be concluded that when $Z_{\mathrm{L}}$ matches $Z_{\text {out }}\left(\omega_{0}\right)$ the coupling is maximised, whereas for any other situation the coupling reduces.

In general, achieving maximum power transfer from the source to the load implies that the source impedance is matched to the load impedance. Analysing the equivalent circuit of Fig. 4 it can be observed that the impedance seen from the source is $Z_{\text {in }}$, which at resonance is given by (5). For example, the impedance seen from the source when the two coils are $50 \mathrm{~mm}$ apart and the load impedance is $50 \Omega$ is about $97 \Omega$. This mismatch will create a large reflection from the load and, although the two coils are very close, only part of the available power will be delivered to the load (Fig. 11). On the other hand, if the distance between the two coils is $80 \mathrm{~mm}$ and the load impedance is $50 \Omega$, the impedance seen from the source is about $46 \Omega$. In this case the mismatch between the source and load is much smaller; therefore more power will be delivered to the load. Once again this situation can be observed graphically in Fig. 11; when $Z_{\text {in }}$ is close to $50 \Omega$ the magnitude of S21 is close to 1 , which is an ideal case, but once $Z_{\text {in }}$ changes because of a change in distance or in load (Fig. 13) the efficiency of the wireless link drops. Therefore, the most desirable condition, when the magnitude of S21 is close to maximum, can be specified as

$$
Z_{\mathrm{s}}=Z_{\text {in }}
$$

Using the above equation and the definition of $Z_{\text {in }}$ given in (5), together with (2), the following relationship for S21 at the resonant frequency can be derived

$$
S_{21}\left(\omega_{0}\right)=\frac{j \omega_{o} L_{12} \sqrt{\left(\left(R_{1}\left(R_{2}+Z_{\mathrm{L}}\right)+\omega_{0}^{2} L_{12}^{2}\right) /\left(R_{2+Z_{\mathrm{L}}}\right)\right) \cdot Z_{\mathrm{L}}}}{R_{1}\left(R_{2}+Z_{\mathrm{L}}\right)+\omega_{0}^{2} L_{12}^{2}}
$$

If the resistances of the two coils $R_{1}$ and $R_{2}$ are very small they can be ignored. Following this assumption (8) can be further simplified to read

$$
S_{21}\left(\omega_{0}\right)=j
$$

It is obvious that the magnitude of $S_{21}$ from the above equation is 1 , hence - according to the definition of the transducer power gain $\left|S_{21}\right|^{2}$ - the ratio of the power delivered to the load over the available power is also 1 .

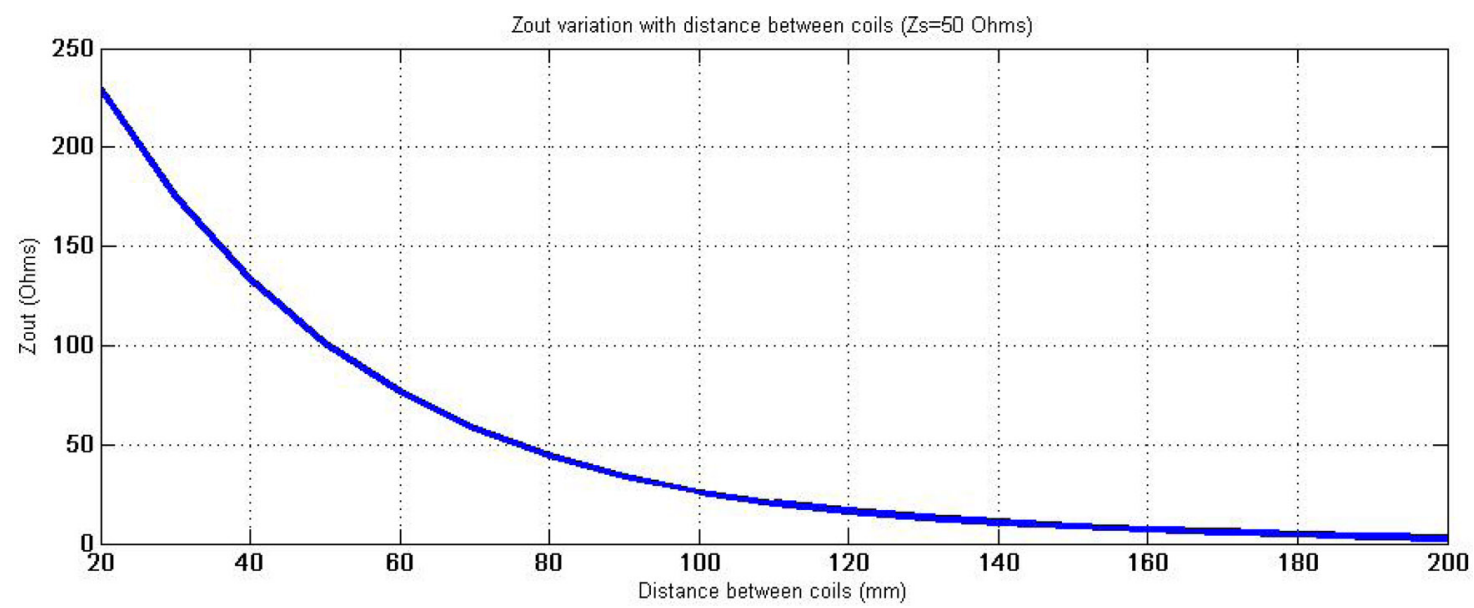

Fig. $12 Z_{\text {out }}\left(\omega_{0}\right)$ variation with the distance between the coils $\left(Z_{S}=50 \Omega\right)$ 


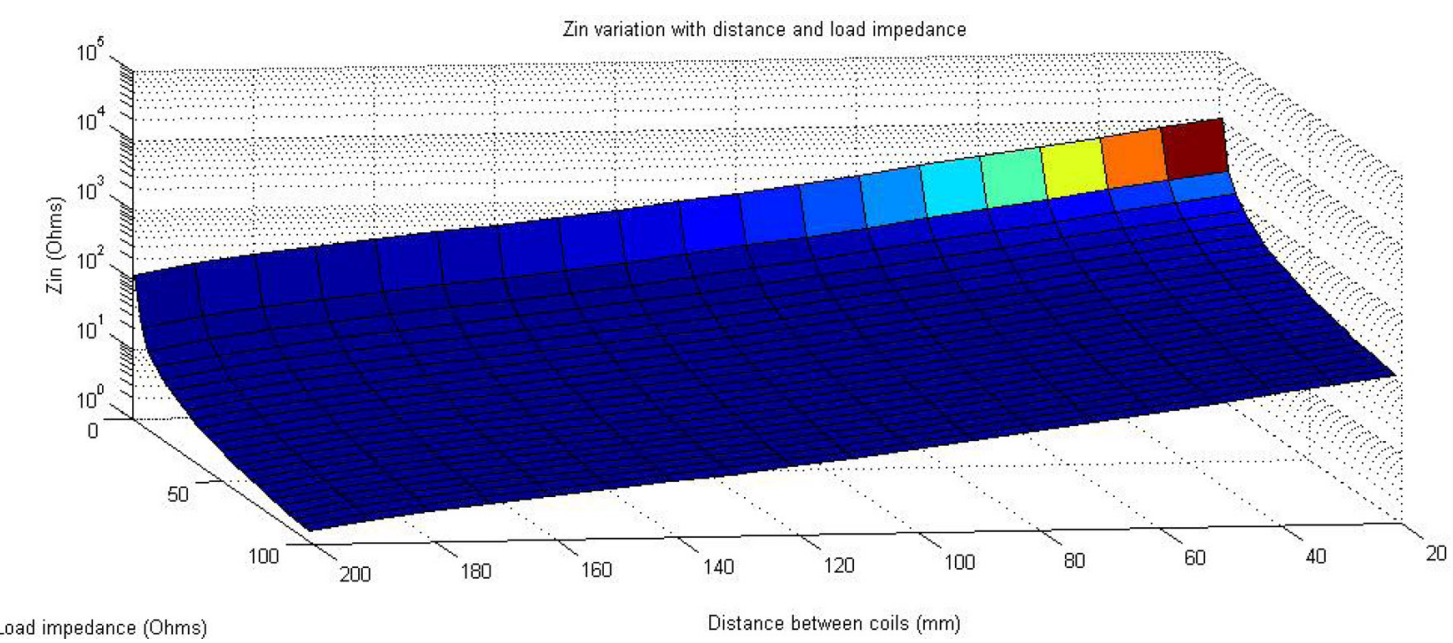

Fig. $13 Z_{\text {in }}\left(\omega_{0}\right)$ variation with the distance between the coils and load impedance

Following the same definition applied to (8) will yield the following result

$$
\left|S_{21}\right|^{2}=\eta_{m}=\frac{Z_{\mathrm{L}} \omega_{0}^{2} L_{12}^{2}}{\omega_{0}^{2} L_{12}^{2}\left(R_{2}+Z_{\mathrm{L}}\right)+R_{1}\left(R_{2}+Z_{\mathrm{L}}\right)^{2}}
$$

which is the same with the efficiency of the WPT system obtained by Kato et al. in [10] using an equivalent circuit approach. However, this result assumes that the source is always matched to the input impedance, which may not be always the case. In a general case, when the source impedance is different from the input impedance, the correct relation between the power delivered to the load with respect to the available power can be inferred from (2) written at the natural resonant frequency

$$
\begin{gathered}
S_{21}\left(\omega_{0}\right)=\frac{2 j \omega L_{12} \cdot \sqrt{Z_{\mathrm{s}} Z_{\mathrm{L}}}}{\left(Z_{\mathrm{s}}+R_{1}\right)\left(Z_{\mathrm{L}}+R_{2}\right)+L_{12}^{2} \omega^{2}} \\
\left|S_{21}\right|^{2}=\eta=\frac{4 Z_{\mathrm{s}} Z_{\mathrm{L}} \omega_{0}^{2} L_{12}^{2}}{\omega_{0}^{2} L_{12}^{2}+\left(R_{1}+Z_{\mathrm{S}}\right)\left(R_{2}+Z_{\mathrm{L}}\right)}
\end{gathered}
$$

It can be shown that (12) may be rewritten in a more convenient form as

$$
\left|S_{21}\right|^{2}=\eta=\eta_{m} \cdot\left(1-\left|\Gamma_{\text {in }}\right|^{2}\right)
$$

where $\Gamma_{\text {in }}$ is the input reflection coefficient defined as

$$
\Gamma_{\text {in }}=\frac{Z_{\text {in }}-Z_{\mathrm{S}}}{Z_{\text {in }}+Z_{\mathrm{S}}}
$$

It can therefore be concluded that if the source impedance matches the input impedance, and the resistances of the two coils are negligible, there will be no reflection and all the power available in the primary coil will be transmitted to the secondary coil. This condition can be achieved for a variety of distances between coils and different loading conditions. Another useful observation is that although matching the source impedance to $Z_{\text {in }}$ is important if the resistances of the two coils are relatively large there will be loss in terms of the wireless link performance. Hence minimising the resistance of the two coils is an important factor when WPT is considered.

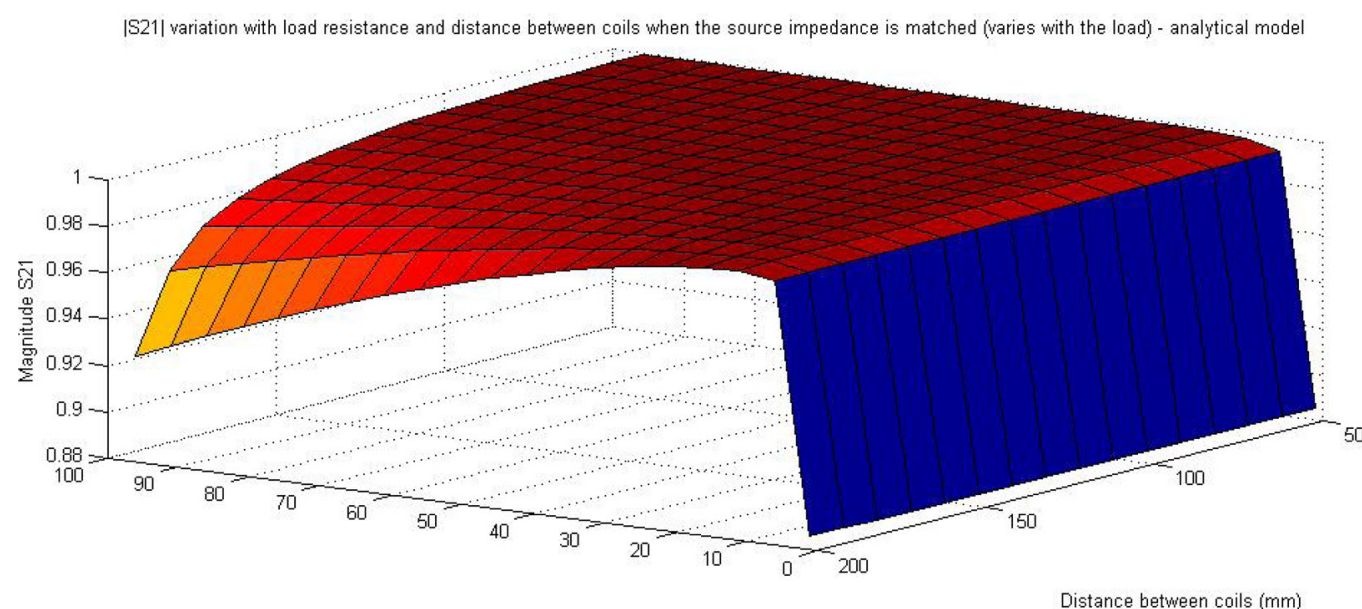

Load resistance (ohms)

Fig. 14 Variation of S21 magnitude when the load and distance between coils is changed (source impedance is matched dynamically with the load and distance) 


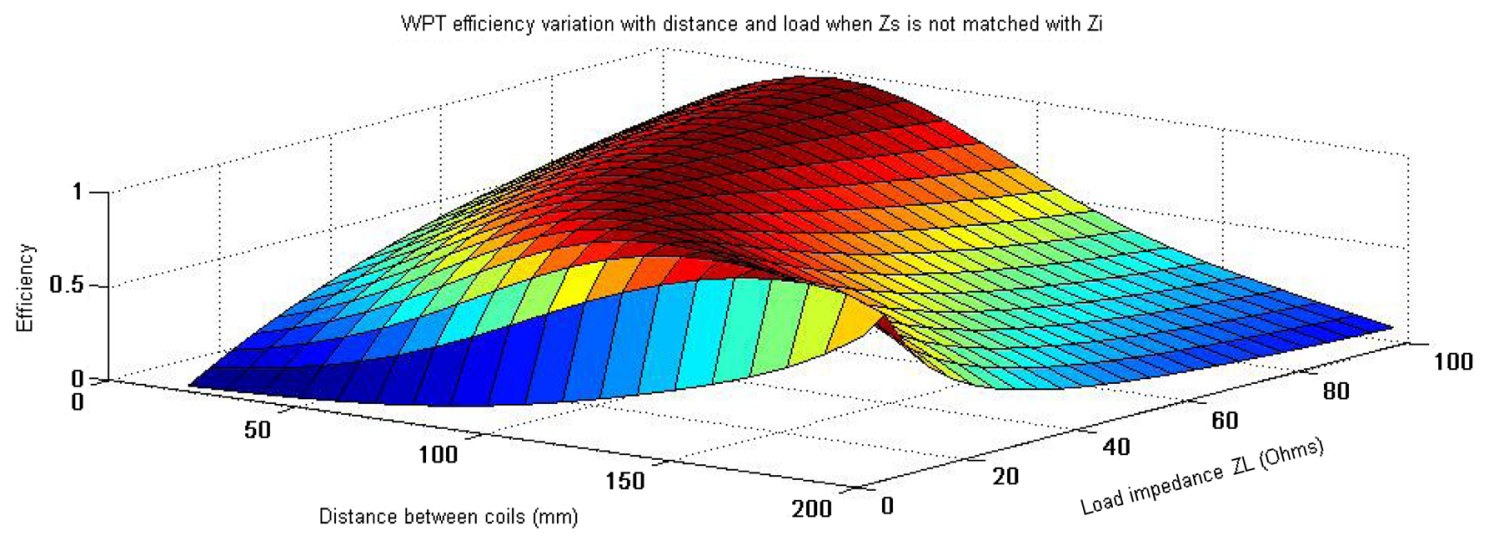

Fig. 15 WPT efficiency variation with distance and load impedance for a fixed source impedance $\left(Z_{S}=50 \Omega\right)$

Fig. 14 shows graphically the improvement in terms of the magnitude of S21 when the source impedance is matched to the input impedance $Z_{\text {in }}$ of the WPT system.

Although the efficiency of the wireless link can be maintained at high values through matching the source to the input impedance, the amount of power that the WPT system can handle is not constant. It will be instructive to analyse again the equivalent circuit of the WPT system presented in Fig. 4 to understand the power handling capability of the WPT at maximum efficiency. One way of

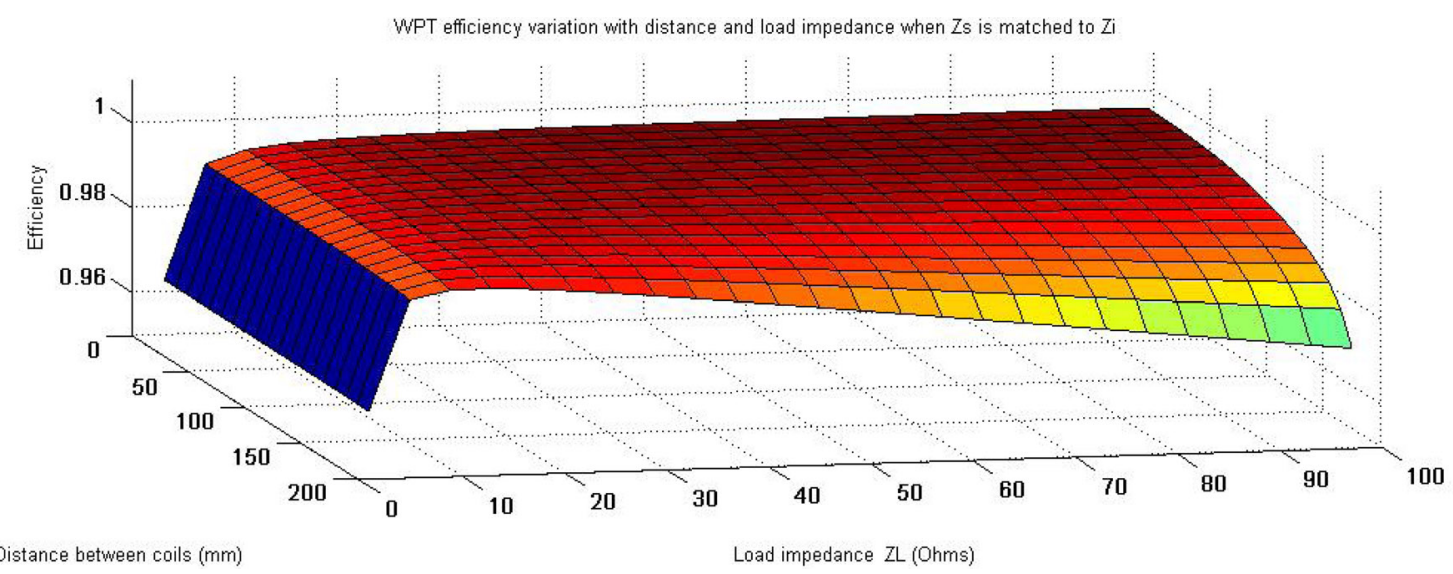

Fig. 16 WPT efficiency variation with distance and load impedance when source impedance is matched to $Z_{\text {in }}$

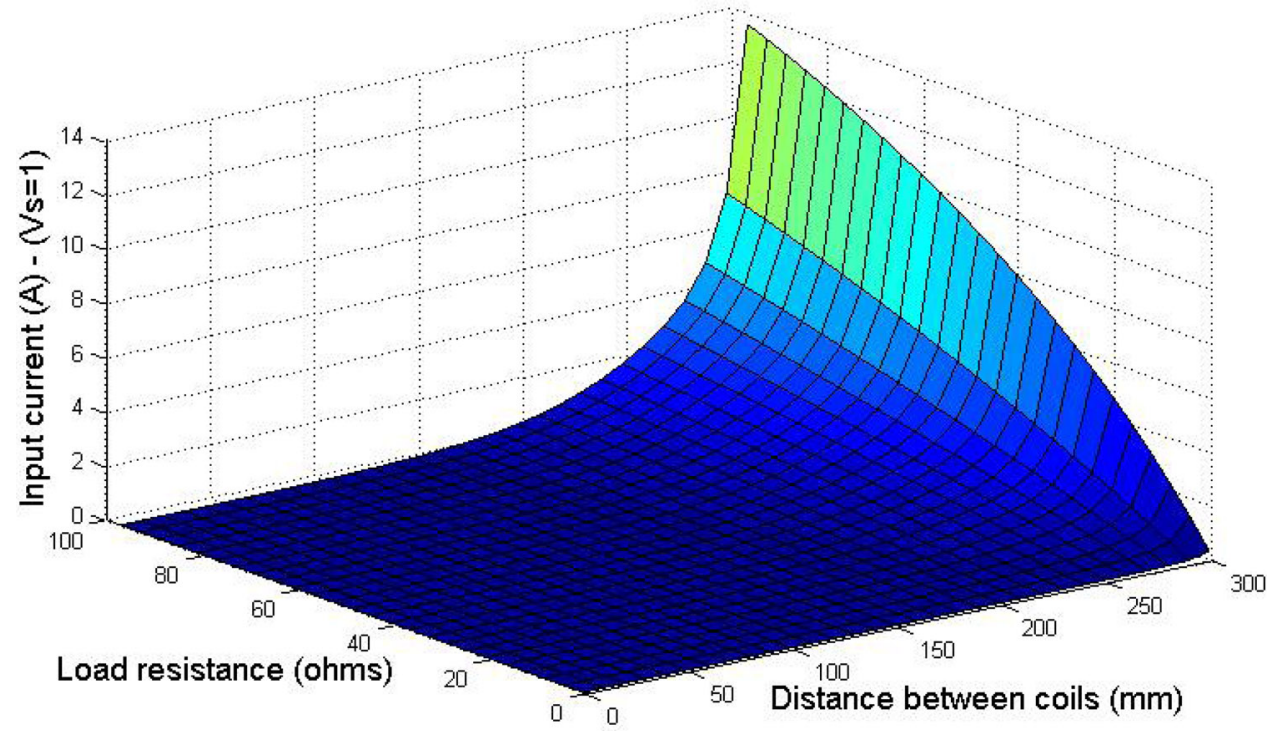

Fig. 17 WPT input current variation for different load conditions and different distances between coils when the source voltage magnitude is $1 \mathrm{~V}$

IET Sci. Meas. Technol., pp. 1-12 doi: 10.1049/iet-smt.2014.0175 


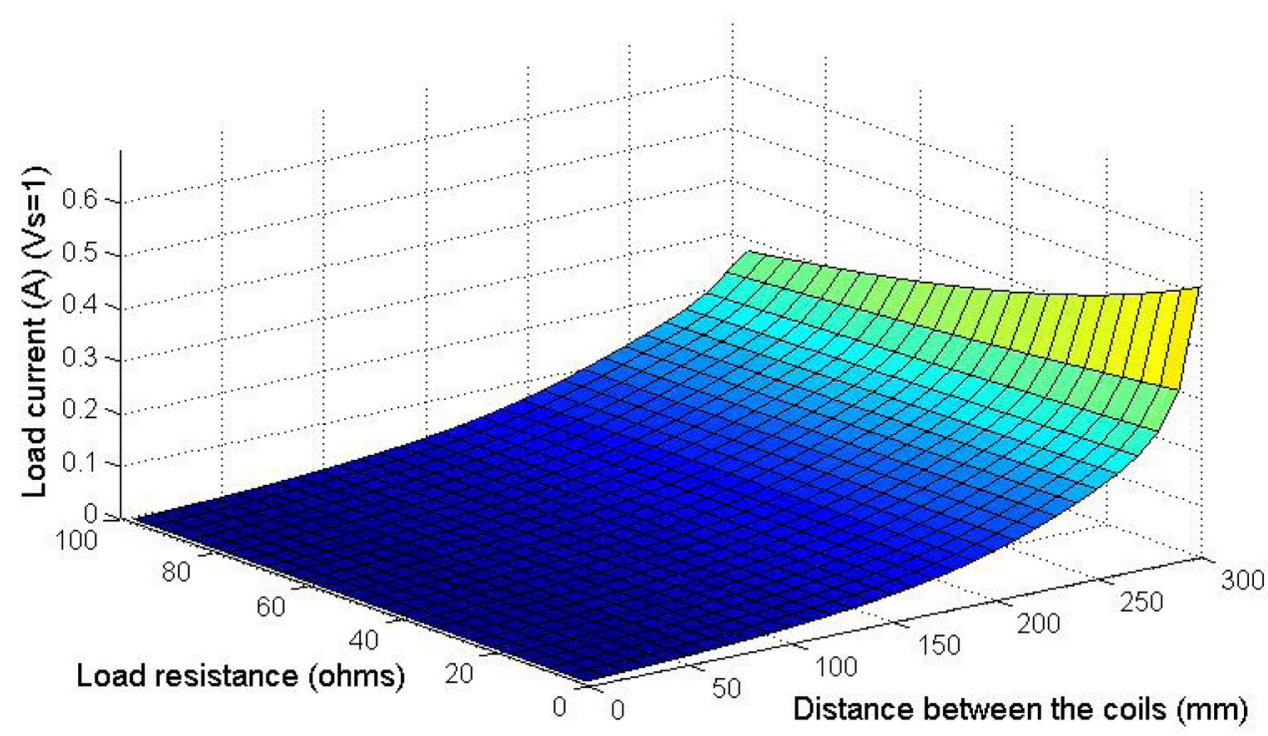

Fig. 18 WPT load current variation for different load conditions and different distances between coils when the source voltage magnitude is $1 \mathrm{~V}$

controlling the impedance seen at the interface between the source and the primary coil is to control the voltage or/and the current at that particular circuit point. Suppose the voltage of the source is constant; then the only way of controlling the impedance is by changing the current. As discussed in the previous paragraph, to achieve high efficiency for the wireless link the source impedance $Z_{\mathrm{s}}$ has to be equal to the input impedance $Z_{\text {in }}$; thus when the input impedance $Z_{\text {in }}$ is large the current coming from the source has to be reduced so that the source impedance $\left(Z_{\mathrm{S}}=\left(V_{s} / I_{\text {in }}\right)\right)$ matches the input impedance. On the other hand, if $Z_{\text {in }}$ is small the current should be increased to match $Z_{\text {in }}$ (see Figs. 15 and 16). Therefore, the power that can be handled by the system at its maximum efficiency condition will be smaller in the case of a large $Z_{\text {in }}$ than in the case of a reduced $Z_{\text {in }}$. According to (6) a small value of $Z_{\text {in }}$ can be achieved either by reducing the coupling between the coils through increasing the distance between them, or by increasing the load resistance. However, both conditions have to be considered with care when maximum efficiency is sought because decreasing the $Z_{\text {in }}$ to maximise the power will force the $Z_{\mathrm{s}}$ to drop and in the limiting case, when either coupling factor is zero or load impedance infinity, the only impedance limiting the current in the primary coil is its own resistance $R_{1}$. As discussed in the previous sections, normally the coil resistance should be minimised leading to a large primary current, which might damage the coil if there is insufficient protection (Fig. 17).

In practical applications the input voltage (source voltage) of the systems is constant; hence it would be useful to understand the variation of all other important parameters of the WPT, such as input current, load current and load voltage, whereas the distance and load vary, as a function of the source voltage. From the equivalent circuit of Fig. 4 it can be noted that the input current can be conveniently written in terms of source voltage and input admittance of the WPT equivalent circuit. At the natural resonance of the

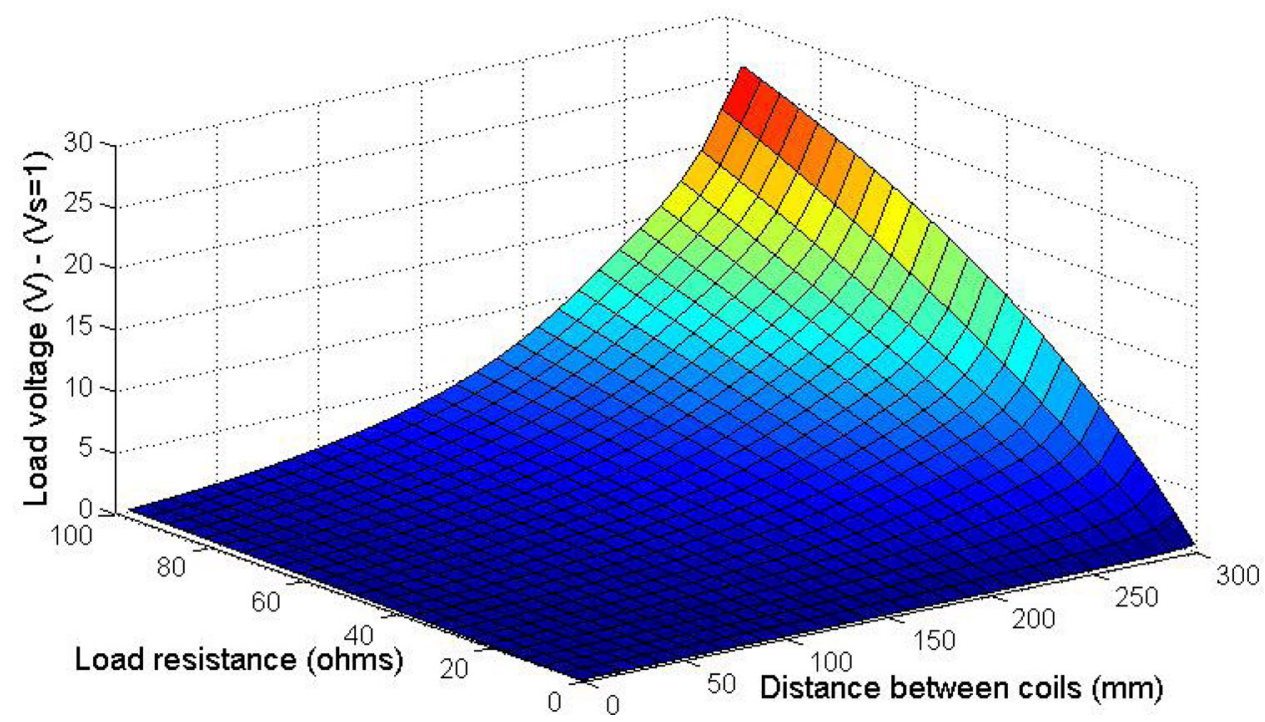

Fig. 19 WPT load voltage variation for different load conditions and different distances between coils when the source voltage magnitude is $1 \mathrm{~V}$ 
two coils the input current is

$$
I_{\text {in }}=V_{s} \cdot Y_{\text {in }}\left(\omega_{0}\right)=\frac{V_{S}}{Z_{\text {in }}\left(\omega_{0}\right)}=V_{S} \cdot \frac{Z_{\mathrm{L}}+R_{2}}{R_{1}\left(Z_{\mathrm{L}}+R_{2}\right)+\left(\omega_{0} L_{12}\right)^{2}}
$$

At resonance the load current can be written in terms of the input current as

$$
I_{\text {load }}=I_{\text {in }} \cdot \frac{j \omega_{0} L_{12}}{Z_{L}+R_{2}}
$$

By substituting (9) into (10) the load current can be written in terms of source voltage as

$$
I_{\text {load }}=V_{S} \cdot Y_{\text {in }}\left(\omega_{0}\right) \cdot \frac{j \omega_{0} L_{12}}{Z_{\mathrm{L}}+R_{2}}
$$

Finally, the load voltage can be written as

$$
V_{\text {load }}=V_{S} \cdot Y_{\text {in }}\left(\omega_{0}\right) \cdot \frac{j \omega_{0} L_{12}}{Z_{\mathrm{L}}+R_{2}} \cdot Z_{\mathrm{L}}
$$

From the above equations it can be seen that the load current will have a phase difference of $90^{\circ}$ with respect to the source current, which in turn is in phase with the source voltage at resonance. Similarly the load voltage has a $90^{\circ}$ phase difference with respect to the source voltage. By plotting the input current, load current and load voltage, for different load conditions and distances between coils, the behaviour of the WPT system can be visualised. Figs. 17-19 show these parameters when the source voltage has an amplitude of $1 \mathrm{~V}$.

It can be observed from Fig. 17 that the input current will be maximised when both the load resistance and the distance are at their largest values. In this case the WPT systems will absorb maximum power. However, this may not be useful as the efficiency of the power transfer is at its lowest under these conditions; at the same time the load voltage is very large (Fig. 18). For the specific case studied here the load voltage could reach values 25 times larger than the source voltage; therefore appropriate control and special insulation have to be considered carefully for such systems.

The maximum load current is obtained when the load resistance is minimum and the distance between the coils is at its highest value (Fig. 17); however, this condition is not very useful from the practical point of view as the input power into the WPT system is small and thus the power delivered to the load will be small too, even if the efficiency of the wireless transfer is high.

\section{Conclusions}

The characteristics of a WPT system have been extracted and studied through simulations verified by measurements conducted on a dedicated experimental rig. From 3D FEM full wave simulations a circuit model of the WPT system has been established. With the help of this circuit model, supported by 3D FEM calculations, the $\mathrm{S}$ parameters of the WPT were extracted. Based on the $\mathrm{S}$ parameters, the efficiency of the WPT system was calculated and analysed. It has been shown that the maximum efficiency is achieved when the source, WPT and the load are simultaneously matched. Further analysis has demonstrated that the efficiency calculated with the help of circuit analysis agrees well with the value obtained from the $\mathrm{S}$ parameter analysis of the WPT system. However, using only the circuit analysis may lead to the wrong conclusion if the source impedance is not carefully considered. The effects on the transfer efficiency of the WPT system, when the load and distance between the coils are changed, have been studied thoroughly. It has been shown that it is possible to maintain the transfer efficiency of the system at a very high level, even for relatively large distance separating the coils, providing simultaneous matching conditions are fulfilled. This means that the input impedance has to be adjusted as the distance and/or load conditions vary. This is particularly important when the distance between the coils is increased.

The mathematical expressions input current, load current and load voltage have been extracted when the source voltage was assumed to be fixed. From the analysis of these equations it has been shown that maximum input power of the system is achieved when the efficiency of system is not at its maximum. Moreover, careful design considerations, including protection, should be applied in such systems, especially for high power applications, as the input current can be very large when the load is large or when the secondary coil is very far. The load voltage may also be very large when the input current is large.

Future work will focus on the effects of massive conducting or shielding structures that may exist in the proximity of practical high power wireless transfer power systems, as well as considering a more realistic source impedance value which will have an influence on the behaviour of the WPT system. Moreover, the implementation of a tapped capacitive divider transformer for matching purposes will be investigated.

\section{References}

1 Elliot, G., Covic, G., Kacprzak, D., Boys, J.T.: 'A new concept: asymmetrically pick-ups for inductively coupled power transfer monorail systems', IEEE Trans. Magn., 2006, 42, (10), pp. 3389-3391

2 Sallan, J., Villa, J.L., Llombart, A., Sanz, J.F.: 'Optimal design of ICPT systems applied of electric vehicle battery charge', IEEE Trans. Ind. Electron., 2009, 56, (6), pp. 2140-2149

3 Wang, C.S., Stielau, O.H., Covic, G.A.: 'Design consideration for contactless electric vehicle battery charger', IEEE Trans. Ind. Electron., 2005, 52, (5), pp. 1308-1313

4 Sergeant, P., Van den Bossche, A.: 'Inductive coupler for contactless power transmission', IET Electr. Power Appl., 2008, 2, (1), pp. 1-7

5 Kurs, A., Karalis, A., Moffatt, R., Joannopoulos, J.D., Fisher, P., Soljacic, M.: 'WPT via strong coupled magnetic resonances', Sci. Express, 2007, 317, (5834), pp. 83-86

6 Chen, L., Liu, S., Zhou, Y.C., Cui, T.J.: 'An optimizable circuit structure for high-efficiency wireless power transfer', IEEE Trans. Ind. Electron., 2013, 60, (1), pp. 339-349

7 Sample, A.P., Meyer, D.A., Smith, J.R.: 'Analysis, experimental results, and range adaptation of magnetically coupled resonators for wireless power transfer', IEEE Trans. Ind. Electron., 2011, 58, (2), pp. 544-554

8 Li, H., Wang, K., Dong, X.: 'Study of efficiency maximization design principles for wireless power transfer system using magnetic resonant coupling'. 2013 IEEE ECCE Asia Downunder, Fifth IEEE Annual Int. Energy Conversion Congress and Exhibition, 2013, pp. 888-892

9 International Commission on Non-Ionizing Radiation Protection ICNIRP Guidelines for limiting exposure to time-varying electric, magnetic and electromagnetic fields (up to $300 \mathrm{GHz}$ )

10 Kato, M., Imura, T., Hori, Y.: 'New characteristics analysis considering transmission distance and load variation in wireless power via magnetic resonant coupling'. 34th IEEE Int. Telecommunications Energy Conf. (INTELECH), 2012 